\title{
TU/e EmonOWEN

\section{Integrated Design of a CVT-equipped Electric Powertrain via Analytical Target Cascading}

\section{Citation for published version (APA):}

Fahdzyana, C. A., Salazar, M., Donkers, T., \& Hofman, T. (2021). Integrated Design of a CVT-equipped Electric Powertrain via Analytical Target Cascading. In 2021 European Control Conference, ECC 2021 (pp. 927-932). Institute of Electrical and Electronics Engineers. https://doi.org/10.23919/ECC54610.2021.9655001

DOI:

10.23919/ECC54610.2021.9655001

Document status and date:

Published: 01/01/2021

\section{Document Version:}

Accepted manuscript including changes made at the peer-review stage

\section{Please check the document version of this publication:}

- A submitted manuscript is the version of the article upon submission and before peer-review. There can be important differences between the submitted version and the official published version of record. People interested in the research are advised to contact the author for the final version of the publication, or visit the $\mathrm{DOI}$ to the publisher's website.

- The final author version and the galley proof are versions of the publication after peer review.

- The final published version features the final layout of the paper including the volume, issue and page numbers.

Link to publication

\section{General rights}

Copyright and moral rights for the publications made accessible in the public portal are retained by the authors and/or other copyright owners and it is a condition of accessing publications that users recognise and abide by the legal requirements associated with these rights.

- Users may download and print one copy of any publication from the public portal for the purpose of private study or research.

- You may not further distribute the material or use it for any profit-making activity or commercial gain

- You may freely distribute the URL identifying the publication in the public portal.

If the publication is distributed under the terms of Article 25fa of the Dutch Copyright Act, indicated by the "Taverne" license above, please follow below link for the End User Agreement:

www.tue.nl/taverne

Take down policy

If you believe that this document breaches copyright please contact us at:

openaccess@tue.nl

providing details and we will investigate your claim. 


\title{
Integrated Design of a CVT-equipped Electric Powertrain via Analytical Target Cascading
}

\author{
Chyannie A. Fahdzyana ${ }^{1}$, Mauro Salazar ${ }^{1}$, Tijs Donkers ${ }^{2}$, Theo Hofman ${ }^{1}$
}

\begin{abstract}
Electric vehicles are gaining momentum as a valid alternative to conventional engine-based cars. In order to meet the high expectation of the market, they must strive for a similar, if not better, performance and driving range. To this end, their powertrain must be carefully designed and account for the interconnections among the various components in an integrated fashion. In this paper, we present a co-design framework for electric powertrains, whereby we jointly optimize the size of the electric machine (EM) and the geometry of a continuously variable transmission (CVT) together with its ratio trajectory, with the goal of minimizing the energy consumption of the vehicle. Specifically, we first frame the minimum-energy co-design problem in an integrated manner, accounting for the CVT geometry and dynamics, and the EM size. Given the problem complexity, we decompose it into an EM-design and a CVT-design subproblem, whereby we jointly optimize the CVTratio trajectory, and leverage analytical target cascading (ATC) to effectively solve the design problem. Finally, we showcase our framework on the New European Driving Cycle (NEDC), highlighting the importance of designing powertrains in an integrated fashion: Compared to the case whereby only the EM, the CVT, or the control are optimized, our joint EM-CVT design can improve the energy consumption of the vehicle by up to $22 \%$.
\end{abstract}

\section{INTRODUCTION}

A S A RESULT of stricter regulations on emissions, electric and hybrid electric vehicles are increasing in popularity [1]. However, in order to fully take effect, such powertrains still have a number of challenges to overcome, namely the initial cost of buying the vehicle and the achievable driving range. One of the components of a vehicle powertrain is the transmission which connects the propulsion source to the wheels. Typically, electric vehicle powertrains are equipped with fixed-gear transmissions. In the past few years, several studies have highlighted the potential energy saving and performance benefits of using multi-speed and continuously variable transmissions (CVTs) in electric vehicle applications [2], [3]. In particular, a CVT is capable of realizing continuous transmission-ratio values, allowing the mover(s) combustion engine and/or electric machine (EM) - to operate at the highest possible efficiency, and improving the driving comfort via a smooth torque transmission. While CVTs typically have lower energy efficiency compared to that of a fixed-gear transmission, their flexibility also enables to downsize the prime mover. This calls for methods to optimize vehicle powertrains in an integrated manner. Against this backdrop, we investigate the problem of jointly optimizing an

\footnotetext{
${ }^{1}$ Control Systems Technology group, Department of Mechanical Engineering, Eindhoven University of Technology, The Netherlands, e-mail: $\{$ c.a.fahdzyana, m.r.u.salazar, t.hofman\}@tue.nl.

2 Control Systems group, Department of Electrical Engineering, Eindhoven University of Technology, The Netherlands, e-mail: m.c.f.donkersetue.nl.
}

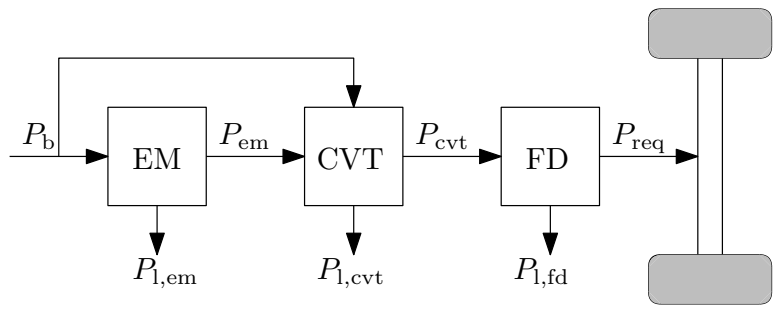

Fig. 1. Schematic diagram of the considered electric powertrain with an electric machine (EM) that supplies the required traction power, and a CVT that is connected to the wheels via a final drive ratio (FD).

electrical powertrain in terms of EM size and CVT geometry, including the ratio control, whilst accounting for the CVT dynamics.

\section{A. Literature Review}

Our work pertains to two research streams, namely, powertrain design and co-design of complex systems. The use of CVTs in conventional and hybrid electric vehicle powertrains has been explored quite extensively [4]-[7]. However, to the best of our knowledge, not much research has been conducted to investigate the benefits of using CVTs in battery electric vehicle powertrain applications. Furthermore, current studies typically employ a simple static CVT model [3], [8], [9], where the dynamic behavior of the system is not considered. In our previous research, we focused on jointly optimizing the geometric design and feedback controller of CVTs [10], also accounting for its low-level dynamics [11], but assuming that the primary mover's (combustion engine) size and operating points (e.g., the rotational speed and torque), as well as the desired ratio trajectory are given.

In general, simultaneously optimizing the plant and control artifacts of complex systems consisting of multiple components may not always be computationally feasible, as it may entail several implementation issues, such as memory limitations and unreasonably high computation times. A possible approach to overcome this issue is to divide the problem into multiple subproblems and solve them via a coordinated optimization strategy converging to the original problem's solution. Traditional system design uses sequential or iterative methods which do not account for the coupling between the separate plant and control problem, and therefore often yield deficient designs. A popular alternative is the nested approach [12]-[14], as it not only guarantees system optimality, but also allows optimal control solution techniques to be directly applied to the control subproblem. Yet this approach is limited by the assumption that an optimal controller does exist for the system [15], which 
may not always be the case, and is based on derivative-free optimization algorithms. Against this backdrop, a number of researchers have studied different decomposition methods for optimal system designs, namely multidisciplinary design optimization [16] and analytical target cascading (ATC) [17]. In particular, ATC has been shown to be a very useful coordination method for decomposed optimization strategies [18], due to its proven convergence properties [19]. However, ATC has not yet been applied to jointly optimize the motor and the transmission of powertrain systems.

Summing up, as far as the authors have ascertained, there is no optimization framework to design electric powertrain systems in an integrated fashion, including the transmission's geometry and control.

\section{B. Statement of Contribution}

To bridge this gap, we present a design optimization framework based on ATC to design a CVT-equipped electric powertrain in an integrated manner. We jointly optimize the size of the EM and the geometry and ratio control of the CVT, accounting for its internal dynamics. We focus on minimizing the electric energy that must be supplied to the EM (e.g., by a battery or a fuel cell) in order to maximize the vehicle's driving range, whilst accounting for the impact of the components' mass on the total power request. Furthermore, we also investigate the benefits of jointly optimizing the powertrain components (i.e., CVT and EM) and their control, as opposed to optimizing only EM and control, CVT and control, or control on its own.

\section{Organization}

This paper is organized as follows: Section II details the models of the powertrain components used in this work. The general co-design problem formulation is presented in Section III, whilst we devise our decomposed optimization framework in Section IV. Finally, we discuss our results in Section V and draw the conclusions in Section VI.

\section{Modeling}

In this study, we consider a simple powertrain configuration shown in Fig. 1. The modeling of the powertrain components of the proposed design problem will be elaborated below.

\section{A. Electric Machine}

We proceed by modeling the EM losses as a continuous function of the motor rotational speed and power. These losses of the EM are also influenced by the size of this component, where they are assumed to scale linearly with the motor size,

$$
P_{\text {loss }, \mathrm{em}}=s_{\mathrm{em}} P_{\text {loss }, \mathrm{em}, \mathrm{o}},
$$

where $s_{\text {em }}$ represents the scaling factor of the motor size, and the original fitted EM loss $P_{\text {loss,em,o }}$ is given by

$$
P_{\text {loss }, \mathrm{em}, \mathrm{o}}=\mathbf{Z}^{\top} \mathbf{Q Z} \text {, }
$$

where $\mathbf{Z}=\left[\begin{array}{lll}1 & \omega_{\mathrm{em}, \mathrm{o}} & P_{\mathrm{em}, \mathrm{o}}\end{array}\right]^{\top}$. We scale the motor with respect to the torque

$$
s_{\mathrm{em}}=\frac{T_{\mathrm{em}}}{T_{\mathrm{em}, \mathrm{o}}},
$$

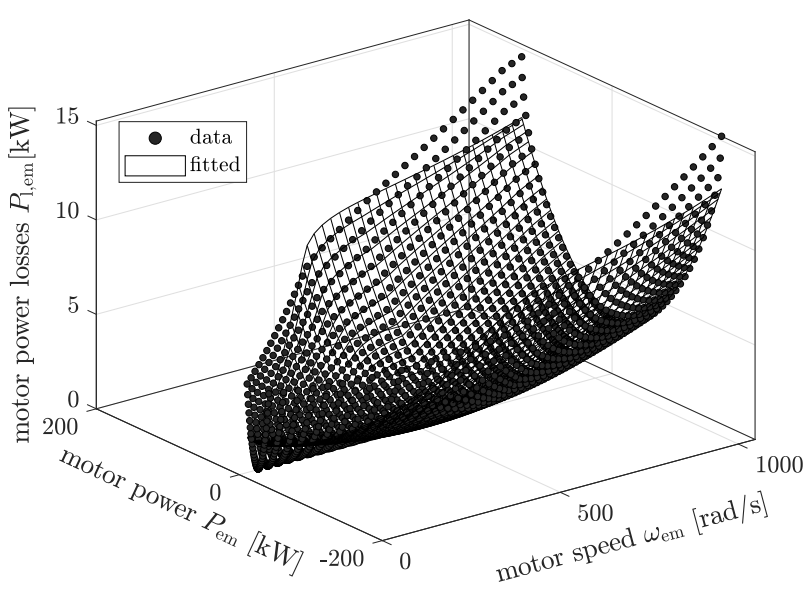

Fig. 2. Fitted EM power loss model considered in this study. The RMSE of the electrical power loss is $4 \%$.

and we obtain a new expression for $P_{\text {loss,em, yielding }}$

$$
P_{\text {loss }, \mathrm{em}}=s_{\mathrm{em}} \cdot \tilde{\mathbf{Z}}^{\top} \mathbf{Q} \tilde{\mathbf{Z}}
$$

where $\tilde{\mathbf{Z}}=\left[\begin{array}{lll}1 & \omega_{\mathrm{em}, \mathrm{o}} & P_{\mathrm{em}} / s_{\mathrm{em}}\end{array}\right]^{\top}, \mathbf{Q}$ is a positive-definite matrix of the motor loss model coefficients. The motor model as a function of the motor speed and power is visualized in Fig. 2. Additionally, the maximum delivered torque of the EM then becomes a function of the scaling parameter $s_{\mathrm{em}}$, given by

$$
\bar{T}_{\mathrm{em}}=s_{\mathrm{em}} \bar{T}_{\mathrm{em}, \mathrm{o}},
$$

where $\bar{T}_{\text {em,o }}$ is a maximum torque (before scaling) that can be delivered by the electric machine. The mass of the EM is expressed as a function of the scaling parameter, such that

$$
M_{\mathrm{em}}=\rho_{\mathrm{em}} s_{\mathrm{em}} \bar{P}_{\mathrm{em}, \mathrm{o}},
$$

where $\rho_{\text {em }}$ is the specific density of the EM in $\mathrm{kg} / \mathrm{kW}$, and $\bar{P}_{\mathrm{em}, \mathrm{o}}$ is the maximum EM power before scaling $\left(s_{\mathrm{em}}=1\right)$.

\section{B. Continuously Variable Transmission}

In this subsection, we discuss the CVT modeling, system dynamics, and the losses considered in this study. Interested readers are referred to [10], [20] for a more detailed explanation of the models. The CVT transmission ratio is defined as the ratio of the output to the input speed,

$$
r_{\mathrm{g}}=\frac{\omega_{\mathrm{s}}}{\omega_{\mathrm{p}}}
$$

where $\omega_{\mathrm{p}}$ and $\omega_{\mathrm{s}}$ are the CVT primary (connected to the electric machine) and secondary rotational speed (connected to the wheels), respectively.

The system dynamics of the CVT transmission is described by the CMM model, derived in [20],

$$
\dot{r}_{\mathrm{g}}=2 \omega_{\mathrm{p}} \Delta \frac{1+\cos ^{2}(\beta)}{\sin (2 \beta)} c\left(r_{\mathrm{g}}\right)\left[\ln \frac{F_{\mathrm{p}}}{F_{\mathrm{s}}}-\left.\ln \frac{F_{\mathrm{p}}}{F_{\mathrm{s}}}\right|_{\mathrm{ss}}\right],
$$

where $\omega_{\mathrm{p}}$ is the rotational speed at the primary side, which is the motor rotational speed $\omega_{\mathrm{em}}, \Delta$ denotes the pulley deformation, $\beta$ is the pulley wedge angle. $F_{\mathrm{p}}$ and $F_{\mathrm{s}}$ are the 
applied primary and secondary clamping forces, and $\left.\frac{F_{\mathrm{p}}}{F_{\mathrm{s}}}\right|_{\mathrm{ss}}$ denotes the steady-state clamping force ratio, which is the ratio needed to sustain $r_{\mathrm{g}}$ such that $\dot{r}_{g}=0$. The term $c\left(r_{\mathrm{g}}\right)$ can be written as

$$
c\left(r_{\mathrm{g}}\right)=c_{1} r_{\mathrm{g}}^{2}+c_{2} r_{\mathrm{g}}+c_{3} .
$$

Furthermore, the steady-state clamping force ratio is described as

$$
\left.\frac{F_{\mathrm{p}}}{F_{\mathrm{s}}}\right|_{\mathrm{ss}}\left(\beta, r_{\mathrm{g}}\right)=a_{1}(\beta) r_{\mathrm{g}}^{2}+a_{2}(\beta) r_{\mathrm{g}}+a_{3}(\beta)+b_{1} \Upsilon^{2}+b_{2} \Upsilon \text {, }
$$

where $\Upsilon$ is given by

$$
\Upsilon=\frac{\left|T_{\mathrm{em}}\right|}{\left|T_{\mathrm{em}}\right|+0.3 \bar{T}_{\mathrm{em}}} .
$$

In addition, the pulley deformation $\Delta$ is described as a function of the secondary clamping force $F_{\mathrm{S}}$,

$$
\begin{aligned}
\Delta\left(F_{\mathrm{s}}\right) & =\left(1+0.02 \cdot\left(F_{\mathrm{s}} / 1000-20\right)\right) \cdot 0.001 \\
F_{\mathrm{s}} & =\frac{\cos (\beta)\left(\left|T_{\mathrm{em}}\right|+0.3 \bar{T}_{\mathrm{em}}\right)}{2 \mu R_{\mathrm{p}}} .
\end{aligned}
$$

We select the CVT control input as $u=$ $\left[\ln \frac{F_{\mathrm{p}}}{F_{\mathrm{s}}}-\left.\ln \frac{F_{\mathrm{p}}}{F_{\mathrm{s}}}\right|_{\mathrm{ss}}\right]$, meaning that the CVT dynamics in (14) can now be written as

$$
\dot{r}_{\mathrm{g}}=2 \omega_{\mathrm{em}} \Delta \frac{1+\cos ^{2}(\beta)}{\sin (2 \beta)} c\left(r_{\mathrm{g}}\right) u .
$$

We aim to optimize the CVT with respect to the physical design as well as its control performance. The physical design parameters considered in this work are the pulley wedge angle $\beta$, shaft radius $R_{1}$ and pulley radius $R_{2}$ [10]. These parameters influence the CVT mass, which is expressed as

$$
M_{\mathrm{cvt}}=M_{\mathrm{cvt}, \mathrm{o}}+M_{\mathrm{var}}\left(\beta, R_{1}, R_{2}, \bar{T}_{\mathrm{em}}\right),
$$

where $M_{\text {cvt,o }}$ is the CVT base mass, $\bar{T}_{\text {em }}$ is the maximum deliverable torque of the EM, and $M_{\mathrm{var}}$ is the variator mass. The variator mass is influenced by the maximum torque and the pulley design parameters, such that

$$
M_{\mathrm{var}}=c_{\mathrm{m}} \bar{T}_{\mathrm{em}}+M_{\mathrm{v}}\left(\beta, R_{1}, R_{2}\right),
$$

where $M_{\mathrm{v}}$ is the pulley and belt mass which is described in [10]. The value of $c_{\mathrm{m}}$ is shown in Table II.

Furthermore, we consider the leakage losses that occur at the pulley hydraulic chambers throughout operation, described as

$$
P_{\text {loss }, \mathrm{cvt}}=c_{\mathrm{p}} p_{\mathrm{p}}^{2}+c_{\mathrm{s}} p_{\mathrm{s}}^{2},
$$

where $c_{\mathrm{p}}$ and $c_{\mathrm{s}}$ are variator leakage factor coefficients obtained from [21]. The CVT losses are directly influenced by the clamping pressures $p_{\mathrm{p}}$ and $p_{\mathrm{s}}$,

$$
\begin{aligned}
& p_{\mathrm{p}}=\frac{F_{\mathrm{p}}-F_{\text {cent }, \mathrm{p}}\left(R_{1}, R_{2}, \omega_{\mathrm{em}}\right)}{A_{\mathrm{p}}\left(R_{1}, R_{2}\right)}, \\
& p_{\mathrm{s}}=\frac{F_{\mathrm{s}}-F_{\text {cent }, \mathrm{s}}\left(R_{1}, R_{2}, \omega_{\mathrm{em}}, r_{\mathrm{g}}\right)}{A_{\mathrm{s}}\left(R_{1}, R_{2}\right)},
\end{aligned}
$$

where $F_{j}$ are the clamping forces, $F_{\text {cent }, j}$ are the centrifugal forces, and $A_{j}$ is the pulley surface area for $j \in\{p, s\}$, and they depend on the geometric parameters of the CVT variator, i.e., the shaft radius $R_{1}$ and the pulley radius $R_{2}$.

\section{Longitudinal vehicle dynamics}

Here, we utilize a backward-facing model to describe the longitudinal dynamics of the vehicle.

$$
\begin{aligned}
F_{\text {roll }} & =M_{\mathrm{veh}} g C_{\mathrm{r}} \cos \left(\alpha_{\text {max }}\right) \\
F_{\text {aero }} & =0.5 \rho_{\mathrm{a}} C_{\mathrm{d}} A_{\mathrm{f}} v_{\mathrm{wh}}^{2} \\
F_{\text {inert }} & =M_{\mathrm{veh}}\left(1+M_{\mathrm{rot}}\right) a_{\mathrm{wh}} \\
F_{\text {grad }} & =M_{\mathrm{veh}} g \sin (\alpha) \\
T_{\mathrm{v}} & =\left(F_{\text {roll }}+F_{\text {aero }}+F_{\text {inert }}+F_{\text {grad }}\right) \cdot R_{\mathrm{wh}} \\
P_{\text {req }} & =T_{\mathrm{v}} \omega_{\mathrm{v}} \\
\omega_{\mathrm{em}} & =\frac{\omega_{\mathrm{v}}}{r_{\mathrm{fd}} r_{\mathrm{g}}}
\end{aligned}
$$

where $F_{\text {roll }}, F_{\text {aero }}, F_{\text {inert }}, F_{\text {grad }}$ are the rolling resistance, aerodynamic drag, inertia, and gradability force, respectively. The vehicle speed $\omega_{\mathrm{v}}$ is given by the drive cycle, and is used to determine the required wheel power $P_{\text {req. }}$. It can be observed that the requested power $P_{\text {req }}$ depends on the mass of the vehicle $M_{\mathrm{veh}}$, which is given by

$$
M_{\mathrm{veh}}=M_{\mathrm{veh}, \mathrm{o}}+M_{\mathrm{em}}+M_{\mathrm{cvt}},
$$

where $M_{\mathrm{veh}, \mathrm{o}}$ is the vehicle base mass, $M_{\mathrm{em}}$ is the EM mass, and $M_{\text {cvt }}$ is the CVT mass.

\section{Co-Design Problem Formulation}

In this section, we present the integrated plant and control design of a battery electric vehicle, taking into account the design of the EM and its corresponding transmission system (CVT). The aim of the optimal design is to minimize the energy along the drivetrain that needs to be supplied by the battery. The combined plant and control design problem is given by

$\min _{\mathbf{x}_{\mathrm{P}}, \mathbf{x}_{\mathrm{C}}} \int_{0}^{t_{f}}\left(P_{\text {req }}\left(\mathbf{x}_{\mathrm{P}}\right)+P_{\text {loss }, \mathrm{em}}\left(\mathbf{x}_{\mathrm{P}}, \mathbf{x}_{\mathrm{C}}\right)+P_{\text {loss }, \mathrm{cvt}}\left(\mathbf{x}_{\mathrm{P}}, \mathbf{x}_{\mathrm{C}}\right)\right) \mathrm{d} t$

where the plant design parameters are the motor scaling parameter and the CVT geometry, $\mathbf{x}_{\mathrm{P}}=\left\{s_{\mathrm{em}}, \beta, R_{1}, R_{2}\right\}$, and the control design parameters are the optimal CVT ratio and input trajectories, $\mathbf{x}_{\mathrm{C}}=\left\{r_{\mathrm{g}}(t), u(t)\right\}$, subject to (9)(14), (17), (20)-(27), as well as

$$
\begin{aligned}
P_{\text {loss }, \mathrm{em}} & =s_{\mathrm{em}}\left[\begin{array}{lll}
1 & \frac{\omega_{\mathrm{v}}}{r_{\mathrm{g}} r_{\mathrm{fd}}} & P_{\mathrm{em}}
\end{array}\right] \mathrm{Q}\left[\begin{array}{c}
1 \\
\frac{\omega_{\mathrm{v}}}{r_{\mathrm{g}} r_{\mathrm{fd}}} \\
P_{\mathrm{em}}
\end{array}\right] \\
P_{\mathrm{em}} & =P_{\mathrm{wh}}+P_{\mathrm{l}, \mathrm{fd}} \\
P_{\mathrm{em}} & =\omega_{\mathrm{em}} T_{\mathrm{em}} \\
P_{\mathrm{em}} & \leq \bar{P}_{\mathrm{em}} \\
\underline{r}_{\mathrm{g}} & \leq r_{\mathrm{g}} \leq \bar{r}_{\mathrm{g}} \\
\underline{s}_{\mathrm{em}} & \leq s_{\mathrm{em}} \leq \bar{s}_{\mathrm{em}} \\
\underline{u} & \leq u \leq \bar{u} \\
\underline{\beta} & \leq \beta \leq \bar{\beta} \\
\underline{R}_{1} & \leq R_{1} \leq \bar{R}_{1} \\
\underline{R}_{2} & \leq R_{2} \leq \bar{R}_{2},
\end{aligned}
$$

where the design parameters are the scalar plant design parameters, including the scaling factor of the EM and the 
CVT geometry, $\mathbf{x}_{\mathrm{P}}=\left\{s_{\mathrm{em}}, \beta, R_{1}, R_{2}\right\}$, as well as the optimized trajectories of the CVT ratio and input signal $\mathbf{x}_{\mathrm{C}}=\left\{r_{\mathrm{g}}(t), u(t)\right\}$.

As observed from the proposed design problem, there are strong dependencies between the plant and control design parameters, objectives, and constraints. Due to this property, solving this integrated powertrain design problem is not a trivial matter. In the subsequent section, we will discuss the proposed deconstructed approach to the co-design problem.

\section{DECOMPOSED FRAMEWORK}

The optimization problem definition proposed in Section III is a highly complex formulation. We therefore propose a decomposed co-design framework based on the concept of ATC to solve the original co-design problem. This approach allows the large co-design problem to be decomposed into smaller subproblems which can be solved iteratively, ensuring that the dependency between the separated subsystems is accounted for.

To proceed in the manner indicated, we first identify the analysis functions' dependencies on the design parameters. We observe that the requested power is influenced by the EM and CVT design parameters; the EM losses depend on the motor scaling parameter and the CVT ratio trajectory; the CVT losses depend on the CVT geometry, as well as the CVT and ratio input trajectory. Based on these relationships, we divide the original optimization problem into two hierarchical subproblems. We first identify the local variablesthe optimized parameters that only appear in the respective subproblems-and the shared variables, which are those that appear in the two or more subproblems. The subproblems optimize for both variable types, while ensuring that the shared variables converge into the same value. In order to do this, we employ a quadratic penalty function in the optimization objective to minimize the discrepancy between the shared variables. The presented framework is explained in more detail below.

\section{A. Upper Level}

We first discretize the original continuous-time formulation using Forward Euler method. The objective of the upper level subproblem is to minimize the requested energy and EM loss, subject to the design parameters, i.e., the motor scaling parameter $\mathbf{x}_{\mathrm{P} 1}=\left[s_{\mathrm{em}}\right]$ and the CVT ratio trajectory $\mathbf{x}_{\mathrm{C} 1}=\left[\mathbf{r}_{\mathrm{g}}\right]$. Mathematically, the optimization formulation in discrete time is given by

$\min _{\mathrm{x}_{\mathrm{P} 1}, \mathrm{x} \mathrm{C} 1} \sum_{k=1}^{N}\left(P_{\mathrm{em}}(k)+P_{\text {loss }, \mathrm{em}}(k)\right) \Delta t+\left(\mathbf{z}^{\mathrm{L}}-\mathbf{z}\right)^{\top} \mathbf{W}\left(\mathbf{z}^{\mathrm{L}}-\mathbf{z}\right)$

subject to (20)-(27) and (30)-(34). Here, $\mathbf{z}$ denotes the vector of shared variables, which in this case are the CVT ratio, EM scaling factor, the CVT mass $\mathbf{z}=$ $\left[r_{\mathrm{g}}(1), r_{\mathrm{g}}(2), \ldots, r_{\mathrm{g}}(N), s_{\mathrm{em}}, M_{\mathrm{cvt}}\right]^{\top}$, and $\mathbf{W}$ is a diagonal matrix of optimization weights $\mathbf{W}=\operatorname{diag}\left(w_{1} \cdot \mathbf{1}, w_{2}, w_{3}\right)$.

As seen from the formulation, the upper-level optimization minimizes the power generated by the EM and the losses, as well as the discrepancy between the shared variables. Furthermore, the optimization also uses several values that are generated by the lower level subproblem, indicated by superscript L. When solving the upper level problem, the parameters with superscript $L$ are treated as constants. Once the optimization at this level is completed, the upper level then propagates the resulting shared variables $\mathrm{z}^{\mathrm{U}}$ to the lower level subproblem as targets.

\section{B. Lower Level}

The lower-level optimization subproblem concerns the minimization of the CVT mass and the leakage losses, as well as the discrepancy between the target (generated by the upperlevel subproblem) and response variables. Mathematically, the optimization of the lower level subproblem is formulated as

$$
\min _{\mathrm{x}_{\mathrm{P} 2}, \mathrm{x}_{\mathrm{C} 2}} \sum_{k=1}^{N} P_{\text {loss }, \mathrm{cvt}}(k) \Delta t+\left(\mathbf{z}^{\mathrm{U}}-\mathbf{z}\right)^{\top} \mathbf{W}\left(\mathbf{z}^{\mathrm{U}}-\mathbf{z}\right),
$$

subject to the constraints described in (9)-(14), (17), (29)-(38). The design parameters are the CVT geometry $\mathbf{x}_{\mathrm{P} 2}=\left[\beta, R_{1}, R_{2}\right]$, as well as the CVT ratio and input trajectory, $\mathbf{x}_{\mathrm{C} 2}=\left[\mathbf{r}_{\mathrm{g}}, \mathbf{u}\right]$. Similarly, the lower level then passes the generated shared variables $\mathbf{z}^{\mathrm{L}}=$ $\left[r_{\mathrm{g}}^{\mathrm{L}}(1), r_{\mathrm{g}}^{\mathrm{L}}(2), \ldots r_{\mathrm{g}}^{\mathrm{L}}(N), s_{\mathrm{em}}^{\mathrm{L}}, M_{\mathrm{cvt}}^{\mathrm{L}}\right]^{\top}$ as a response to the upper level, and a new iteration begins. The iterative process is repeated until the termination criterion $\epsilon$ is reached, given by

$$
\begin{aligned}
& \frac{1}{N} \sum_{k=1}^{N}\left(\frac{r_{\mathrm{g}}^{\mathrm{U}}(k)-r_{\mathrm{g}}^{\mathrm{L}}(k)}{r_{\mathrm{g}}^{\mathrm{U}}(k)}\right)^{2} \cdot \Delta t+\left(\frac{s_{\mathrm{em}}^{\mathrm{U}}-s_{\mathrm{em}}^{\mathrm{L}}}{s_{\mathrm{em}}^{\mathrm{U}}}\right)^{2} \\
& +\left(\frac{M_{\mathrm{cvt}}^{\mathrm{U}}-M_{\mathrm{cvt}}^{\mathrm{L}}}{M_{\mathrm{cvt}}^{\mathrm{U}}}\right)^{2} \leq \epsilon_{\mathrm{tol}, j},
\end{aligned}
$$

where $\epsilon_{\mathrm{tol}, j}$ is the maximum allowable tolerance on the discrepancies between the shared variables.

By selecting $r_{\mathrm{g}}, s_{\mathrm{em}}$, and $M_{\mathrm{cvt}}$ as the shared variables, the original subproblems can be decomposed into smaller and more manageable tasks, while ensuring that the strategy converges to a tractable set of solutions.

\section{RESUlts AND Discussion}

This section presents the results obtained with the proposed co-design formulation over the New European Driving Cycle (NEDC). The decomposed optimization subproblems are parsed with CasAdi [22] and solved in Python using the IPOPT solver [23] provided by the OPTI toolbox. Specifically, we compare our integrated design and control optimization results whereby the EM's size, the CVT's geometry and the ratio trajectory are jointly optimized, to the results stemming from the sole optimization of (i) EM's size and ratio trajectory, (ii) CVT's geometry and ratio trajectory, and (iii) ratio trajectory. The results are summarized in Table I, whilst the simulation parameters used in this study can be found in Table II.

It can be seen from Fig. 3 that at the start of the iterative process, the discrepancy $\epsilon$ between the shared variables of the subproblems is large. However, it gradually decreases in the next iterations until $\epsilon$ reaches an acceptable value. Consequently, we observe that both the upper and lower level optimization subproblems of the decomposition-based 
TABLE I

RESULTS OF USING THE PROPOSED DECOMPOSITION BASED CO-DESIGN APPROACH

\begin{tabular}{|c|c|c|c|c|c|c|}
\hline Solution & Description & Optimize CVT+EM & Optimize CVT only & Optimize EM only & Optimize control only & Unit \\
\hline$s_{\mathrm{em}}$ & Motor scaling & 0.6 & 1 & 0.6 & 1 & - \\
\hline$\beta$ & Wedge angle & 7.14 & 9.16 & 11 & 11 & deg \\
\hline$R_{1}$ & Shaft radius & 20 & 20 & 23.5 & 23.5 & $\mathrm{~mm}$ \\
\hline$R_{2}$ & Pulley radius & 88.5 & 88.5 & 85.5 & 85.5 & $\mathrm{~mm}$ \\
\hline$M_{\mathrm{cvt}}$ & CVT mass & 65.23 & 93.53 & 93.6 & 93.6 & $\mathrm{~kg}$ \\
\hline$E_{1, \mathrm{em}}$ & EM loss & 0.76 & 1.26 & 0.76 & 1.26 & MJ \\
\hline$E_{l, c y t}+E_{l f d}$ & CVT loss & 61.9 & 67.94 & 63.8 & 69.6 & $\mathrm{~kJ}$ \\
\hline$E_{\mathrm{b}}$ & Motor input energy & 1.97 & 2.51 & 1.99 & 2.52 & MJ \\
\hline
\end{tabular}

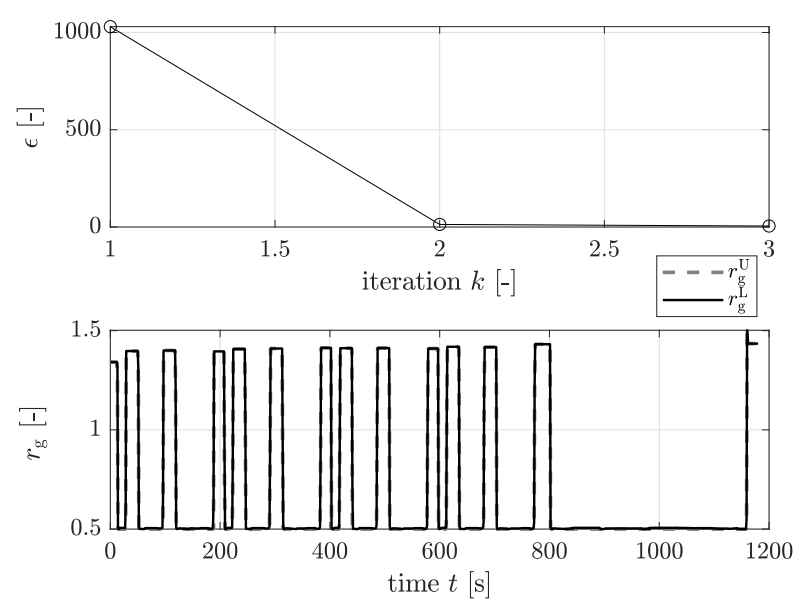

Fig. 3. Evolution of $\epsilon$ per iteration for the proposed decomposition-based optimization.

optimization framework manage to converge to the same optimized ratio trajectory $r_{\mathrm{g}}$. Additionally, although we can observe in Fig. 3 that the proposed decomposed optimization eventually converges, there exists no guarantee on both the optimality of the obtained results and the convergence of the algorithm due to the nonlinearity of the optimization problem.

Furthermore, we observe that optimizing both the CVT and EM as well as the ratio trajectory yields the lowest energy consumption, whilst solely optimizing CVT design and/or control yields a significantly higher energy consumption, which can be explained as follows: Table I exemplifies that the overall losses are dominated by the EM, which explains the higher energy consumption for the case studies whereby the EM's size is not optimized. We can observe that the resulting ratio trajectories for the case of optimizing only the control or the CVT parameters do not differ significantly from each other, as shown in Fig. 4.

Moreover, in Fig. 5, depicting the optimal power supplied to the EM for the different case studies, we do not find a big difference in optimizing the CVT and/or the ratio trajectory, which can be ascribed to the fact that the CVT's baseline design is already good enough for a relatively mild driving cycle like the NEDC. However, the power can be substantially reduced when both the EM and CVT are concurrently optimized. Finally, we underline the fact that optimizing the powertrain design and control in an integrated

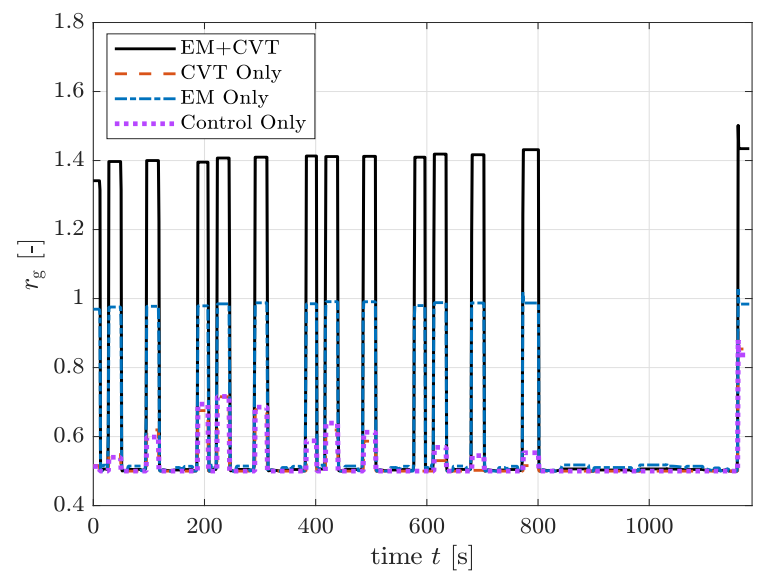

Fig. 4. Resulting optimized ratio trajectory over a representative drive cycle (NEDC) for the different case studies in this paper.
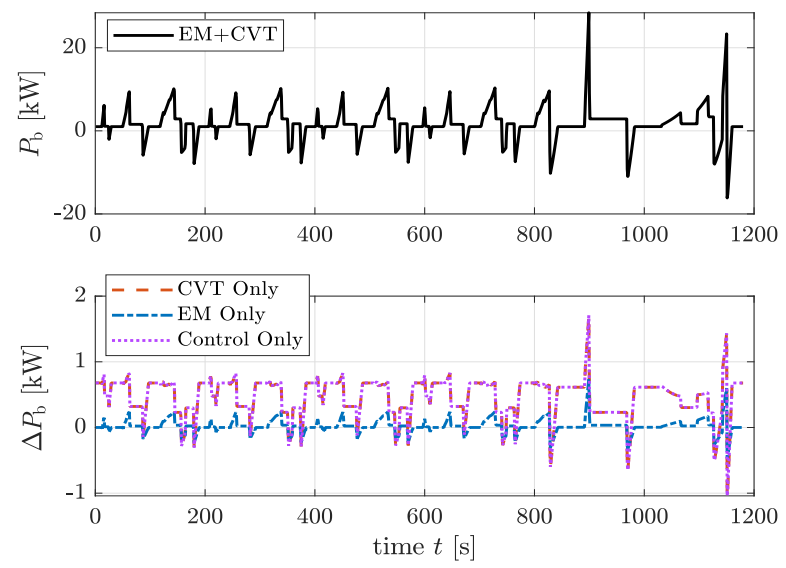

Fig. 5. Resulting instantaneous battery power $P_{\mathrm{b}}$ over a representative drive cycle (NEDC) for the different case studies considered in this paper. The upper plot displays the required battery power throughout the cycle for the case of joint EM-CVT plant and control design. The lower plot depicts the differences of battery requested power $\Delta P_{\mathrm{b}}$ obtained for the remaining case studies with respect to that of the EM-CVT design. It can be observed that jointly optimizing the EM, CVT, and the control strategy yields the lowest required battery energy.

manner can improve the energy consumption up to $22 \%$.

\section{CONCLUSIONS}

In this paper, we have explored a decomposed co-design approach to optimize a CVT-equipped powertrain in terms 
TABLE II

SIMULATION PARAMETERS

\begin{tabular}{|l|l|r|l|}
\hline Parameters & Description & Values & Unit \\
\hline $\bar{r}_{\mathrm{g}}$ & overdrive ratio & 2.5 & - \\
$\underline{r}_{\mathrm{g}}$ & underdrive ratio & 0.45 & - \\
$\bar{\beta}_{\mathrm{R}}$ & min. wedge angle & 7 & $\mathrm{deg}$ \\
$\bar{R}_{1}$ & min. shaft radius & 20 & $\mathrm{~mm}$ \\
$\overline{\bar{\beta}}_{2}$ & min. pulley radius & 85.5 & $\mathrm{~mm}$ \\
$\bar{R}_{1}$ & max. wedge angle & 13 & $\mathrm{deg}$ \\
$\bar{R}_{2}$ & max. shaft radius & 30 & $\mathrm{~mm}$ \\
$\epsilon_{\mathrm{tol}, 1}$ & max. pulley radius & 88.5 & $\mathrm{~mm}$ \\
$\epsilon_{\mathrm{tol}, 2}$ & tolerance on $\epsilon_{1}$ & 1 & - \\
$\rho_{\mathrm{em}}$ & tolerance on $\epsilon_{2}$ & 10 & $\mathrm{~kg}$ \\
$T_{\mathrm{max}, \mathrm{o}}$ & EM specific density & 0.9 & $\mathrm{~kg} / \mathrm{kW}$ \\
$\rho_{\mathrm{a}}$ & air density & 305 & $\mathrm{Nm}$ \\
$C_{\mathrm{d}}$ & drag resistance & 1.23 & $\mathrm{~kg} / \mathrm{m}$ \\
$A_{\mathrm{f}}$ & frontal area & 0.35 & - \\
$R_{\mathrm{wh}}$ & wheel radius & 2.38 & $\mathrm{~m}{ }^{2}$ \\
$M_{\mathrm{rot}}$ & vehicle rotational mass factor & 0.35 & $\mathrm{~m}$ \\
$M_{\mathrm{veh}, \mathrm{o}}$ & vehicle base mass & 1000 & $\mathrm{~kg}$ \\
$M_{\mathrm{cvt}, \mathrm{o}}$ & CVT base mass & 50 & $\mathrm{~kg}$ \\
$c_{\mathrm{m}}$ & CVT mass model coefficient & 0.24 & $\mathrm{~kg} / \mathrm{Nm}$ \\
$w_{1}$ & optimization weight & 300 & - \\
$w_{2}$ & optimization weight & 100 & - \\
$w_{3}$ & optimization weight & 100 & $1 / \mathrm{kg}$ \\
$\Delta t$ & discretization time & 1 & $\mathrm{~s}$ \\
\hline & & \\
\hline
\end{tabular}

of electric motor (EM) sizing and CVT geometry and control. Specifically, we have leveraged analytical target cascading (ATC) to dissect the original nonlinear system design problem into smaller and more tractable optimization subproblems. We have shown the benefits of optimizing the design and control of the electric powertrain's components in an integrated manner, underlining the strong interconnection between the plant design (component sizes) and the control design (performance, energy consumption). Finally, our results revealed that, whilst the CVT baseline design is already good enough for the NEDC, jointly optimizing the EM can significantly improve the energy consumption of the vehicle, highlighting the potential of designing future automotive systems in an integrated manner and prompting more detailed studies.

In the future, we would like to improve the performance of our decomposed co-design framework and expand our design problem to include a battery and/or a fuel cell, and more complex architectures. Moreover, we want to apply the proposed framework to more aggressive scenarios, e.g., employing more demanding acceleration profiles. Finally, we are interested in comparing the performance achievable with different transmission technologies such as fixed-gear and multi-speed transmissions.

\section{ACKNOWLEDGEMENT}

The authors would like to thank Dr. Ilse New for proofreading this paper, and Dr. Ir. Pascal Etman for his useful advice.

\section{REFERENCES}

[1] IEA, “Global ev outlook 2020," IEA, Paris, Tech. Rep., 2020.

[2] P. Walker, S. Abdul Rahman, B. Zhu, and N. Zhang, "Modelling, simulations, and optimisation of electric vehicles for analysis of transmission ratio selection," Advances in Mechanical Engineering, vol. 5, 2013.
[3] T. Hofman and M. Salazar, "Transmission ratio design for electric vehicles via analytical modeling and optimization," in IEEE Vehicle Power and Propulsion Conference, 2020, in press.

[4] R. Pfiffner and L. Guzzella, "Optimal operation of cvt-based powertrains," Int. Journal of Robust and Nonlinear Control, vol. 11, no. 11, pp. 1003-1021, 2001.

[5] T. Kim and H. Kim, "Performance of integrated engine-cvt control considering powertrain loss and cvt response lag," Journal of Automobile Engineering, vol. 216, no. 7, 2002.

[6] N. Murgovski, L. M. Johannesson, and B. Egardt, "Optimal battery dimensioning and control of a cvt phev powertrain," IEEE Transactions on Vehicular Technology, vol. 63, no. 5, pp. 2151-2161, 2014.

[7] C. Mayet, J. Welles, A. Bouscayrol, T. Hofman, and B. LemaireSemail, "Influence of a cvt on the fuel consumption of a parallel medium-duty electric hybrid truck," Mathematics and Computers in Simulation, vol. 158, pp. 120-129, 2019.

[8] F. J. R. Verbruggen, M. Salazar, M. Pavone, and T. Hofman, "Joint design and control of electric vehicle propulsion systems," in European Control Conference, 2020.

[9] O. Borsboom, C. A. Fahdzyana, T. Hofman, and M. Salazar, "A convex optimization framework for minimum lap time design and control of electric race cars," IEEE Transactions on Vehicular Technology, 2020, submitted.

[10] C. A. Fahdzyana, M. Salazar, and T. Hofman, "Integrated plant and control design for a continuously variable transmission," IEEE Transactions on Vehicular Technology, 2020, conditionally accepted.

[11] _ "A decomposed co-design strategy for continuously variable transmission design," in American Control Conference, 2021, in press.

[12] E. Silvas, E. Bergshoeff, T. Hofman, and M. Steinbuch, "Comparison of bi-level optimization frameworks for sizing and control of a hybrid electric vehicle," in IEEE Vehicle Power and Propulsion Conference, 2014.

[13] M. Pourabdollah, E. Silvas, N. Murgovski, M. Steinbuch, and B. Egardt, "Optimal sizing of a series phev: Comparison between convex optimization and particle swarm optimization," in IFAC Workshop on Engine and Powertrain Control, Simulation and Modeling, 2015.

[14] Z. Ma, N. Murgovski, B. Egardt, and S. Cu, "Comprehensive analysis and optimal configurations of the evt powertrain," IEEE Transactions on Vehicular Technology, vol. 68, no. 10, pp. 9573 - 9587, 2019.

[15] J. Reyer, "Combined embodiment design and control optimization: Effects of cross-disciplinary coupling," Ph.D. dissertation, Univ. of Michigan, 2000.

[16] P. W. Jansen, R. E. Perez, and J. Martins, "Aerostructural optimization of nonplanar lifting surfaces," AIAA Journal of Aircraft, vol. 47, no. 5, pp. 1490-1503, 2010.

[17] Y. Kim, H, D. G. Rideout, P. Y. Papalambros, and J. Stein, "Analytical target cascading in automotive vehicle design," ASME Journal of Mechanical Design, vol. 125, no. 3, pp. 481-489, 2003.

[18] S. Tosserams, L. F. P. Etman, P. Y. Papalambros, and J. E. Rooda, "An augmented lagrangian relaxation for analytical target cascading using the alternating direction method of multipliers," Structural and Multidisciplinary Optimization, vol. 31, pp. 176-189, 2006.

[19] N. Michelena, H. Park, and P. Papalambros, "Convergence properties of analytical target cascading," AIAA Journal, vol. 41, no. 5, p. 897-905, 2012.

[20] G. Carbone, L. Mangialardi, B. Bonsen, C. Tursi, and P. A. Veenhuizen, "Cvt dynamics: Theory and experiments," Mechanism and Machine Theory, vol. 42, no. 4, pp. 409-428, 2007.

[21] S. van der Meulen, "High-performance control of continuously variable transmissions," Ph.D. dissertation, Eindhoven University of Technology, 2010.

[22] J. A. E. Andersson, J. Gillis, G. Horn, J. B. Rawlings, and M. Diehl, "Casadi - a software framework for nonlinear optimization and optimal control," Mathematical Programming Computation, vol. 11, no. 1, pp. 1-36, 2019.

[23] A. Wächter and L. Biegler, "On the implementation of a primaldual interior point filter line search algorithm for large-scale nonlinear programming," Mathematical Programming, vol. 106, no. 1, pp. 2557, 2006. 